\title{
Cliques in Graphs Excluding a Complete Graph Minor
}

\author{
David R. Wood* \\ School of Mathematical Sciences \\ Monash University \\ Melbourne, Australia \\ david. wood@monash.edu
}

Submitted: Nov 17, 2015; Accepted: Jul 16, 2016; Published: Aug 5, 2016

Mathematics Subject Classifications: 05C83, 05C35, 05C69

\begin{abstract}
This paper considers the following question: What is the maximum number of $k$-cliques in an $n$-vertex graph with no $K_{t}$-minor? This question generalises the extremal function for $K_{t}$-minors, which corresponds to the $k=2$ case. The exact answer is given for $t \leqslant 9$ and all values of $k$. We also determine the maximum total number of cliques in an $n$-vertex graph with no $K_{t}$-minor for $t \leqslant 9$. Several observations are made about the case of general $t$.
\end{abstract}

Keywords: graph theory, minor, clique

\section{Introduction}

A basic question of extremal graph theory asks: for a class $\mathcal{G}$ of graphs, what is the maximum number of edges in an $n$-vertex graph in $\mathcal{G}$ ? The answer is called the extremal function for $\mathcal{G}$. Consider the following two classical examples. Turán's Theorem [34] says that every $n$-vertex graph with no $K_{t}$-subgraph has at most $\left(\frac{t-2}{2 t-2}\right) n^{2}$ edges, with equality only for the complete $(t-1)$-partite graph with $\frac{n}{t-1}$ vertices in each colours class (called the Turán graph). And Euler's formula implies that the maximum number of edges in a planar graph with $n \geqslant 3$ vertices equals $3 n-6$.

One way to generalise these results is to consider cliques instead of edges. A clique in a graph is a set of pairwise adjacent vertices. A $k$-clique is a clique of cardinality $k$. Since a 2-clique is simply an edge, the following natural generalisations of the above question arise: For a class $\mathcal{G}$ of graphs,

* Research supported by the Australian Research Council. 
- what is the maximum number of $k$-cliques in an $n$-vertex graph in $\mathcal{G}$, and

- what is the maximum number of cliques in an $n$-vertex graph in $\mathcal{G}$ ?

Let cliques $(G)$ be the number of cliques in a graph $G$. Let cliques $(G, k)$ be the number of $k$-cliques in a graph $G$. Of course, $\operatorname{cliques}(G, 0)=1, \operatorname{cliques}(G, 1)=|V(G)|$ and $\operatorname{cliques}(G, 2)=|E(G)|$.

Zykov [36] generalised Turán's Theorem by answering the above questions for the class of graphs with no $K_{t}$-subgraph. He proved that for $t>k \geqslant 0$, every graph with $n \geqslant k$ vertices and no $K_{t}$-subgraph contains at most $\left(\begin{array}{c}t-1 \\ k\end{array}\right)\left(\frac{n}{t-1}\right)^{k}$ cliques of size $k$, and every graph with $n$ vertices and no $K_{t}$-subgraph contains at most $\left(\frac{n}{t-1}+1\right)^{t-1}$ cliques. Both bounds are tight for the Turán graph. Bounds on the number of $k$-cliques in graphs of given maximum degree have been extensively studied $[1,6,12,17,18,35]$. Several papers have established upper bounds on the number of $k$-cliques in terms of the number of vertices and the number of edges, or more generally, in terms of the number of $(\leqslant k-1)$-cliques $[5,10,11,16,29]$.

For planar graphs, Hakimi and Schmeichel [19] proved that the maximum number of triangles is $3 n-8$, and Wood [35] proved that the maximum number of 4 -cliques is $n-3$, and in total the maximum number of cliques is $8 n-16$. See [27] for earlier upper bounds for planar graphs and see [9] for an extension to arbitrary surfaces.

This paper considers these questions in graph classes defined by an excluded minor, thus generalising the above results for planar graphs. This direction has been recently pursued by several authors $[13,14,23,26,28]$. These works have focused on asymptotic results when the excluded minor is a general complete graph $K_{t}$. The primary focus of this paper is exact results, when the excluded minor is $K_{3}, K_{4}, K_{5}, K_{6}, K_{7}, K_{8}$ or $K_{9}$ (Sections 3-5). We also make several observations and conjectures about general $K_{t}$-minor-free graphs (Sections 6-7).

While bounds on the number of cliques in minor-closed classes are of independent interest, such results have had diverse applications, including the asymptotic enumeration of minor-closed classes [26], and in the analysis of an algorithm for finding small separators [28], which in turn has been applied to finding shortest paths [31] and in matrix sparsification [2] for example.

Let cliques $(n, t, k)$ be the maximum number of $k$-cliques in a $K_{t}$-minor-free graph on $n$ vertices. Let cliques $(n, t)$ be the maximum number of cliques in a $K_{t}$-minor-free graph on $n$ vertices. Of course, if $n \leqslant t-1$ then $K_{n}$ is $K_{t}$-minor-free, in which case

$$
\operatorname{cliques}(n, t, k)=\left(\begin{array}{l}
n \\
k
\end{array}\right) \text { and } \operatorname{cliques}(n, t)=2^{n} \text {. }
$$

The following example provides an important lower bound on $\operatorname{cliques}(n, t, k)$. For an integer $\ell \geqslant 1$, an $\ell$-tree is a graph defined recursively as follows. First, the complete graph $K_{\ell}$ is an $\ell$-tree. Then, if $C$ is an $\ell$-clique in an $\ell$-tree, then the graph obtained by adding a new vertex adjacent only to $C$, is also a an $\ell$-tree. Every $\ell$-tree has tree-width at most $\ell$, and thus contains no $K_{\ell+2}$-minor. Observe that for every $\ell$-tree $G$ with $n$ vertices, 
cliques $(G, k)=\left(\begin{array}{c}\ell \\ k-1\end{array}\right)\left(n-\frac{(\ell+1)(k-1)}{k}\right)$ and cliques $(G)=2^{\ell}(n-\ell+1)$. Hence for $n \geqslant t-2$ and $t>k \geqslant 1$,

$$
\begin{aligned}
\operatorname{cliques}(n, t, k) & \geqslant\left(\begin{array}{l}
t-2 \\
k-1
\end{array}\right)\left(n-\frac{(k-1)(t-1)}{k}\right) \\
\operatorname{cliques}(n, t) & \geqslant 2^{t-2}(n-t+3) .
\end{aligned}
$$

The results of this paper show that these lower bounds hold with equality for many values of $t$ and $k$. This is the case for $n \in\{t-2, t-1\}$ by (1).

The starting point for our investigation is the following classical result by Dirac [8] for $t \in\{3,4,5\}$ and by Mader [24] for $t \in\{6,7\}$.

Theorem $1([8,24])$. For $t \in\{3,4,5,6,7\}$, the maximum number of edges in a $K_{t}$-minorfree graph on $n \geqslant t-2$ vertices satisfies

$$
\operatorname{cliques}(n, t, 2)=(t-2)\left(n-\frac{t-1}{2}\right) .
$$

Mader [24] observed that Theorem 1 does not hold with $t=8$. Let $K_{c \times 2}$ be the complete $c$-partite graph $K_{2,2, \ldots, 2}$ with $n=2 c$ vertices, which can be thought of as $K_{2 c}$ minus a perfect matching. In the $t=8$ case, Theorem 1 would give a bound of $6 n-21$ on the number of edges in a $K_{8}$-minor-free graph, whereas Mader [24] observed that $K_{2,2,2,2,2}$ has $n=10$ vertices, $40>6 \cdot 10-21$ edges, and contains no $K_{8}$-minor. $K_{c \times 2}$ will be an important example throughout this paper. In general, Wood [35] proved that $K_{c \times 2}$ contains no $K_{t}$-minor where $t=\left\lfloor\frac{3}{2} c\right\rfloor+1$, and a $K_{t-1}$-minor in $K_{c \times 2}$ is obtained from a $K_{c}$ subgraph by contracting a $\left\lfloor\frac{c}{2}\right\rfloor$-edge matching in the remaining graph.

The following theorems summarise the main contributions of this paper.

Theorem 2. For $t \in\{3,4, \ldots, 9\}$ and $k \in\{1,2, \ldots, t-1\}$ and $n \geqslant t-2$,

$$
\operatorname{cliques}(n, t, k)=\left(\begin{array}{l}
t-2 \\
k-1
\end{array}\right)\left(n-\frac{(k-1)(t-1)}{k}\right),
$$

except for $(t, k) \in\{(8,2),(9,2),(9,3)\}$ and certain values of $n$ (made precise below) in which case

$$
\operatorname{cliques}(n, t, k)=\left(\begin{array}{l}
t-2 \\
k-1
\end{array}\right)\left(n-\frac{(k-1)(t-1)}{k}\right)+1 .
$$

The case $k=2$ (that is, number of edges) was established by Dirac [8] for $t \in\{3,4,5\}$, by Mader [24] for $t \in\{6,7\}$, by Jørgensen [20] for $t=8$, and by Song and Thomas [30] for $t=9$. Note that our proof depends on the case $k=2$ and does not reprove the existing results.

For the total number of cliques, we prove:

Theorem 3. For $t \in\{3,4,5,6,7,8,9\}$, the maximum number of cliques in a $K_{t}$-minorfree graph on $n \geqslant t-2$ vertices satisfies

$$
\operatorname{cliques}(n, t)=2^{t-2}(n-t+3) \text {. }
$$

We employ the following notation. For a vertex $v$ in a graph $G$, let $N(v)$ be the set of neighbours of $v$, let $N[v]:=N(v) \cup\{v\}$, let $G(v):=G[N(v)]$, and let $G[v]:=G[N[v]]$. 


\section{Cockades}

This section introduces a well known construction that will be important later. Let $H$ be a graph containing a $k$-clique. An $(H, k)$-cockade is defined recursively as follows. First, $H$ is an $(H, k)$-cockade. And if $H_{1}$ and $H_{2}$ are $(H, k)$-cockades, then the graph obtained from pasting $H_{1}$ and $H_{2}$ on a $k$-clique is an $(H, k)$-cockade. It is easy to count cliques in cockades.

Lemma 4. For $i \in\{1,2\}$, let $G_{i}$ be a graph with $n_{i}$ vertices such that $\operatorname{cliques}\left(G_{i}, k\right)=$ $a\left(n_{i}-r\right)+\left(\begin{array}{l}r \\ k\end{array}\right)$, for some fixed $a$ and $r \geqslant k$. Let $G$ be obtained by pasting $G_{1}$ and $G_{2}$ on an $r$-clique. Then $G$ has $n=n_{1}+n_{2}-r$ vertices, and cliques $(G, k)=a(n-r)+\left(\begin{array}{l}r \\ k\end{array}\right)$.

Proof.

$$
\begin{aligned}
\operatorname{cliques}(G, k) & =\operatorname{cliques}\left(G_{1}, k\right)+\operatorname{cliques}\left(G_{2}, k\right)-\left(\begin{array}{l}
r \\
k
\end{array}\right) \\
& =a\left(n_{1}-r\right)+\left(\begin{array}{l}
r \\
k
\end{array}\right)+a\left(n_{2}-r\right)+\left(\begin{array}{l}
r \\
k
\end{array}\right)-\left(\begin{array}{l}
r \\
k
\end{array}\right) \\
& =a\left(n_{1}+n_{2}-2 r\right)+\left(\begin{array}{l}
r \\
k
\end{array}\right) \\
& =a(n-r)+\left(\begin{array}{l}
r \\
k
\end{array}\right) .
\end{aligned}
$$

Lemma 5. For $i \in\{1,2\}$, let $G_{i}$ be a graph with $n_{i}$ vertices such that $\operatorname{cliques}\left(G_{i}\right)=$ $a\left(n_{i}-r\right)+2^{r}$, for some fixed $a$ and $r \geqslant 0$. Let $G$ be obtained by pasting $G_{1}$ and $G_{2}$ on an $r$-clique. Then $G$ has $n=n_{1}+n_{2}-r$ vertices, and cliques $(G)=a(n-r)+2^{r}$.

Proof.

$$
\begin{aligned}
\operatorname{cliques}(G) & =\operatorname{cliques}\left(G_{1}\right)+\operatorname{cliques}\left(G_{2}\right)-2^{r} \\
& =\left(a\left(n_{1}-r\right)+2^{r}\right)+\left(a\left(n_{2}-r\right)+2^{r}\right)-2^{r} \\
& =a\left(n_{1}+n_{2}-2 r\right)+2^{r} \\
& =a(n-r)+2^{r} .
\end{aligned}
$$

Lemma 6. For every $\left(K_{c \times 2}, c\right)$-cockade $G$ on $n$ vertices and for $k \in\{0,1, \ldots, c\}$,

$$
\begin{aligned}
\operatorname{cliques}(G, k) & =\frac{1}{c}\left(\begin{array}{l}
c \\
k
\end{array}\right)\left(2^{k}-1\right)(n-c)+\left(\begin{array}{l}
c \\
k
\end{array}\right), \text { and } \\
\operatorname{cliques}(G) & =\frac{1}{c}\left(3^{c}-2^{c}\right)(n-c)+2^{c} .
\end{aligned}
$$

Proof. The first claim follows from Lemma 4 with $r=c$ and $a=\frac{1}{c}\left(\begin{array}{l}c \\ k\end{array}\right)\left(2^{k}-1\right)$ since cliques $\left(K_{c \times 2}, k\right)=\left(\begin{array}{l}c \\ k\end{array}\right) 2^{k}=a(2 c-c)+\left(\begin{array}{l}c \\ k\end{array}\right)$. The second claim follows from Lemma 5 with $r=c$ and $a=\frac{1}{c}\left(3^{c}-2^{c}\right)$ since cliques $\left(K_{c \times 2}\right)=3^{c}=a(2 c-c)+2^{c}$. 


\section{$3 \quad K_{t}$-minor-free graphs with $t \leqslant 7$}

This section proves Theorems 2 and 3 for $t \leqslant 7$.

Theorem 7. For $t \in\{3,4,5,6,7\}$ and $k \in\{1,2, \ldots, t-1\}$ and $n \geqslant t-2$, the maximum number of $k$-cliques in a $K_{t}$-minor-free graph on $n$ vertices satisfies

$$
\operatorname{cliques}(n, t, k)=\left(\begin{array}{l}
t-2 \\
k-1
\end{array}\right)\left(n-\frac{(k-1)(t-1)}{k}\right) .
$$

Proof. The lower bound is provided by (2). For the upper bound, we proceed by induction on $n+k$. The claim is trivial if $k=1$. Theorem 1 proves the claim when $k=2$. Now assume that $k \geqslant 3$. In the case $n=t-2$ the claimed upper bound on cliques $(n, t, k)$ is $\left(\begin{array}{l}n \\ k\end{array}\right)$, which obviously holds. Let $G$ be a $K_{t}$-minor-free graph on $n \geqslant t-1$ vertices.

First suppose that $\operatorname{deg}(v) \leqslant t-2$ for some vertex $v$ of $G$. Each clique of $G$ either contains $v$ or does not contain $v$. The $k$-cliques of $G$ that contain $v$ are in 1-1 correspondence with the $(k-1)$-cliques of $G(v)$. And the cliques of $G$ that do not contain $v$ are exactly the cliques of $G-v$. Thus

$$
\operatorname{cliques}(G, k)=\operatorname{cliques}(G(v), k-1)+\operatorname{cliques}(G-v, k),
$$

which by induction is at most

$$
\left(\begin{array}{c}
\operatorname{deg}(v) \\
k-1
\end{array}\right)+\left(\begin{array}{c}
t-2 \\
k-1
\end{array}\right)\left(n-1-\frac{(k-1)(t-1)}{k}\right) \leqslant\left(\begin{array}{c}
t-2 \\
k-1
\end{array}\right)\left(n-\frac{(k-1)(t-1)}{k}\right),
$$

as desired.

Now assume that $G$ has minimum degree at least $t-1$. For each $k$-clique $C$ in $G$ send a charge of $\frac{1}{k}$ to each vertex in $C$. The charge received by each vertex $v$ equals $\frac{1}{k}$ cliques $(G(v), k-1)$. Since the total charge equals cliques $(G, k)$, and $G(v)$ is $K_{t-1}$-minorfree,

$$
\operatorname{cliques}(G, k)=\frac{1}{k} \sum_{v \in V(G)} \operatorname{cliques}(G(v), k-1) \leqslant \frac{1}{k} \sum_{v \in V(G)} \operatorname{cliques}(\operatorname{deg}(v), t-1, k-1) .
$$

(This argument is essentially that of Fomin et al. [13, Lemma 5].) Since $\operatorname{deg}(v) \geqslant t-1$, by induction,

$$
\begin{aligned}
\operatorname{cliques}(G, k) & \leqslant \frac{1}{k} \sum_{v \in V(G)}\left(\begin{array}{l}
t-3 \\
k-2
\end{array}\right)\left(\operatorname{deg}(v)-\frac{(k-2)(t-2)}{k-1}\right) \\
& =\frac{1}{k}\left(\begin{array}{l}
t-3 \\
k-2
\end{array}\right)\left(2|E(G)|-\frac{(k-2)(t-2) n}{k-1}\right) \\
& \leqslant \frac{1}{k}\left(\begin{array}{l}
t-3 \\
k-2
\end{array}\right)\left(2 \operatorname{cliques}(n, t, 2)-\frac{(k-2)(t-2) n}{k-1}\right) .
\end{aligned}
$$


By Theorem 1,

$$
\begin{aligned}
\operatorname{cliques}(G, k) & \leqslant \frac{1}{k}\left(\begin{array}{l}
t-3 \\
k-2
\end{array}\right)\left(2(t-2)\left(n-\frac{t-1}{2}\right)-\frac{(k-2)(t-2) n}{k-1}\right) \\
& =\frac{(t-2)}{k}\left(\begin{array}{l}
t-3 \\
k-2
\end{array}\right)\left(2 n-(t-1)-\frac{(k-2) n}{k-1}\right) \\
& =\frac{(t-2)}{k}\left(\begin{array}{l}
t-3 \\
k-2
\end{array}\right)\left(\left(\frac{k}{k-1}\right) n-(t-1)\right) \\
& =\frac{(t-2)}{(k-1)}\left(\begin{array}{l}
t-3 \\
k-2
\end{array}\right)\left(n-\frac{(k-1)(t-1)}{k}\right) \\
& =\left(\begin{array}{l}
t-2 \\
k-1
\end{array}\right)\left(n-\frac{(k-1)(t-1)}{k}\right),
\end{aligned}
$$

as desired.

Theorem 8. For $t \in\{3,4,5,6,7\}$, the maximum number of cliques in a $K_{t}$-minor-free graph on $n \geqslant t-2$ vertices equals $2^{t-2}(n-t+3)$.

Proof. The lower bound is (3). The upper bound follows from Theorem 7 since

$$
1+\sum_{k=1}^{t-1}\left(\begin{array}{l}
t-2 \\
k-1
\end{array}\right)\left(n-\frac{(k-1)(t-1)}{k}\right)=2^{t-2}(n-t+3) .
$$

Theorem 7 and 8 were previously proved for $t=5$ by Wood [35] (using a different method).

\section{$4 \quad K_{8}$-minor-free graphs}

Determining the maximum number of cliques in a $K_{8}$-minor-free graph is more difficult than in the $t \leqslant 7$ cases, since $K_{2,2,2,2,2}$ would be a counterexample to Theorem 1 with $t=8$ (see Section 1). In fact, every $\left(K_{2,2,2,2,2}, 5\right)$-cockade would be a counterexample. Jørgensen [20] showed these are the only counterexamples.

Theorem 9 ([20]). Every $K_{8}$-minor-free graph on $n \geqslant 6$ vertices has at most $6 n-21$ edges or is a $\left(K_{2,2,2,2,2}, 5\right)$-cockade (which has $6 n-20$ edges).

We now prove Theorem 2 for $K_{8}$-minor-free graphs with $k \geqslant 3$.

Theorem 10. For $k \in\{3,4,5,6,7\}$ the maximum number of $k$-cliques in a $K_{8}$-minor-free graph on $n \geqslant 6$ vertices satisfies

$$
\operatorname{cliques}(n, 8, k)=\left(\begin{array}{c}
6 \\
k-1
\end{array}\right)\left(n-\frac{7(k-1)}{k}\right) .
$$


Proof. The lower bound is provided by (2). For the upper bound, let $G$ be a $K_{8}$-minorfree graph on $n \geqslant 7$ vertices. We proceed by induction on $n$ with $k$ fixed. In the base case with $n \in\{6,7\}$, the result holds by (1). Now assume that $n \geqslant 8$.

Say $G$ is a $\left(K_{2,2,2,2,2}, 5\right)$-cockade. Then $\operatorname{cliques}(G, 6)=\operatorname{cliques}(G, 7)=0$. By Lemma 6 , $\operatorname{cliques}(G, 3)=14 n-60 \leqslant 15 n-70($ since $n \geqslant 10)$ and cliques $(G, 4)=15 n-70 \leqslant 20 n-105$ and cliques $(G, 5)=\frac{31}{5} n-30 \leqslant 15 n-84$. This show that cliques $(G, k) \leqslant\left(\begin{array}{c}6 \\ k-1\end{array}\right) n-\left(\begin{array}{l}7 \\ k\end{array}\right)(k-1)$. Now assume that $G$ is not a $\left(K_{2,2,2,2,2}, 5\right)$-cockade. Thus $|E(G)| \leqslant 6 n-21$ by Theorem 9 .

The remainder of the proof is analogous to the proof of Theorem 7 , so we sketch it briefly. First, delete a vertex of degree at most 5 and apply induction. Now assume minimum degree at least 6 . Charge each $k$-clique to its vertices, and count the charge at each vertex $v$ with respect to $\operatorname{deg}(v)$ and the number of $(k-1)$-cliques in $G(v)$, which is $K_{7}$-minor-free (applying Theorem 7 ). Counting the total charge, (4) gives

$$
\operatorname{cliques}(G, k) \leqslant \frac{1}{k}\left(\begin{array}{c}
5 \\
k-2
\end{array}\right)\left(2|E(G)|-\frac{(k-2) 3 n}{k-1}\right) .
$$

Since $|E(G)| \leqslant 6 n-21$, it follows by the same analysis used in the proof of Theorem 7 that cliques $(G, k) \leqslant\left(\begin{array}{c}6 \\ k-1\end{array}\right)\left(n-\frac{7(k-1)}{k}\right)$.

Theorem 11. The maximum number of cliques in a $K_{8}$-minor-free graph on $n \geqslant 6$ vertices equals $64(n-5)$.

Proof. Lemma 6 implies that a $\left(K_{2,2,2,2,2}, 5\right)$-cockade is far from extremal for the total number of cliques (since $\frac{211}{5} n-179<64(n-5)$ ). The result then follows from Theorem 9 and Theorem 10, with 6-trees providing the extremal example (see (2)).

\section{$5 \quad K_{9}$-minor-free graphs}

Song and Thomas [30] determined the extremal function for $K_{9}$-minors and characterised the extremal examples.

Theorem 12 (Song and Thomas [30]). Every $K_{9}$-minor-free graph on $n \geqslant 7$ vertices has at most $7 n-28$ edges or is a $\left(K_{1,2,2,2,2,2}, 6\right)$-cockade or is isomorphic to $K_{2,2,2,3,3}$.

Essentially the same method used above determines the maximum number of $k$-cliques in a $K_{9}$-minor-free graph for $k \geqslant 4$.

Theorem 13. For $k \in\{4,5,6,7,8\}$, the maximum number of $k$-cliques in a $K_{9}$-minorfree graph on $n \geqslant 7$ vertices equals

$$
\left(\begin{array}{c}
7 \\
k-1
\end{array}\right)\left(n-\frac{8(k-1)}{k}\right)
$$

Proof. The lower bound is provided by (2). We proceed by induction on $n$ with $k$ fixed. In the base case with $n \in\{7,8\}$, the result holds by (1). Let $G$ be a $K_{9}$-minor-free graph on $n \geqslant 9$ vertices. 
Say $G$ is a $\left(K_{1,2,2,2,2,2}, 6\right)$-cockade. Since $\operatorname{cliques}\left(K_{1,2,2,2,2,2}, 4\right)=1 \cdot\left(\begin{array}{l}5 \\ 3\end{array}\right) \cdot 2^{3}+\left(\begin{array}{l}5 \\ 4\end{array}\right) 2^{4}=160$, by Lemma 4 with $r=6$ and $k=4$ we have cliques $(G, 4)=29(n-6)+\left(\begin{array}{l}6 \\ 4\end{array}\right)<35(n-6)=$ $\left(\begin{array}{c}7 \\ k-1\end{array}\right)\left(n-\frac{8(k-1)}{k}\right)$. Since cliques $\left(K_{1,2,2,2,2,2}, 5\right)=1 \cdot\left(\begin{array}{l}5 \\ 4\end{array}\right) \cdot 2^{4}+2^{5}=112$, by Lemma 4 with $r=6$ and $k=5$ we have cliques $(G, 5)=\frac{106}{5}(n-6)+6<35\left(n-\frac{32}{5}\right)=\left(\begin{array}{c}7 \\ k-1\end{array}\right)\left(n-\frac{8(k-1)}{k}\right)$. Since cliques $\left(K_{1,2,2,2,2,2}, 6\right)=2^{5}=32$, by Lemma 4 with $r=k=6$ we have cliques $(G, 6)=$ $\frac{31}{5}(n-6)+1 \leqslant 21\left(n-\frac{20}{3}\right)=\left(\begin{array}{c}7 \\ k-1\end{array}\right)\left(n-\frac{8(k-1)}{k}\right)$. For $k \in\{7,8\}$ we have cliques $(G, k)=0$.

If $G \cong K_{2,2,2,3,3}$, then cliques $(G, 4)=3 \cdot 2^{2} \cdot 3^{2}+2 \cdot 2^{3} \cdot 3=156<210=\left(\begin{array}{l}7 \\ 3\end{array}\right)\left(12-\frac{8 \cdot 3}{4}\right)$ and cliques $(G, 5)=2^{3} \cdot 3^{2}<196=\left(\begin{array}{l}7 \\ 4\end{array}\right)\left(12-\frac{8 \cdot 4}{5}\right)$. For $k \in\{6,7,8\}$ we have cliques $(G, k)=0$.

We may now assume that $G$ is not a $\left(K_{1,2,2,2,2,2}, 6\right)$-cockade and $G \nsubseteq K_{2,2,2,3,3}$. By Theorem $12,|E(G)| \leqslant 7 n-28$.

The remainder of the proof is analogous to the proof of Theorem 7 , so we sketch it briefly. First, delete a vertex of degree at most 6 and apply induction. Now assume minimum degree at least 7 . Charge each $k$-clique to its vertices, and count the charge at each vertex $v$ with respect to $\operatorname{deg}(v)$ and the number of $(k-1)$-cliques in $G(v)$, which is $K_{8}$-minor-free (applying Theorem 10 since $k-1 \geqslant 3$ ). Counting the total charge, (4) gives

$$
\operatorname{cliques}(G, k) \leqslant \frac{1}{k}\left(\begin{array}{c}
6 \\
k-2
\end{array}\right)\left(2|E(G)|-\frac{(k-2)(7) n}{k-1}\right)
$$

Since $|E(G)| \leqslant 7 n-28$, it follows by the same analysis used in the proof of Theorem 7 that cliques $(G, k) \leqslant\left(\begin{array}{c}7 \\ k-1\end{array}\right)\left(n-\frac{8(k-1)}{k}\right)$.

With a bit more work, we now determine the maximum number of triangles in a $K_{9}$-minor-free graph.

Theorem 14. Every $K_{9}$-minor-free graph on $n \geqslant 7$ vertices contains at most $21 n-112$ triangles, except for $K_{1,2,2,2,2,2}$ which has $120=21 n-111$ triangles.

Proof. We proceed by induction on $n \geqslant 7$. Let $G$ be a $K_{9}$-minor-free graph with $n$ vertices. Assume the result holds for such graphs with less than $n$ vertices. If $n=7$ then $G$ contains at most $\left(\begin{array}{l}7 \\ 3\end{array}\right)=35=21 \cdot 7-112$ triangles. Now assume that $n \geqslant 8$.

Case 1 . $G \cong K_{2,2,2,3,3}$ : Then $n=12$ and $G$ has $134<21 n-112$ triangles. Now assume that $G \approx K_{2,2,2,3,3}$

Case 2. $G \cong K_{1,2,2,2,2,2}$ : Then $G$ contains $\left(\begin{array}{l}5 \\ 3\end{array}\right) 2^{3}=80$ triangles that avoid the dominant vertex, and 40 triangles that include the dominant vertex. Thus $G$ contains $120=21$. $11-111$ triangles, as claimed. Now assume that $G \neq K_{1,2,2,2,2,2}$.

Case 3. $G$ is a $\left(K_{1,2,2,2,2,2}, 6\right)$-cockade: Then $n>11$ as otherwise Case 2 applies. Using the calculation in Case 2, it follows from Lemma 4 that $G$ contains $20 n-100$ triangles, which is less than $21 n-111$ since $n>11$. Now assume that $G$ is not a $\left(K_{1,2,2,2,2,2}, 6\right)$ cockade.

Case 4. $\operatorname{deg}(v) \leqslant 7$ for some vertex $v$ in $G$ : First suppose that $G-v \not K_{1,2,2,2,2,2}$. By induction, $G-v$ contains at most $21(n-1)-112$ triangles. The number of triangles that include $v$ equals $|E(G(v))|$, which is at most $\left(\begin{array}{l}7 \\ 2\end{array}\right)=21$. In total, $G$ contains at most $21 n-112$ triangles. Now assume that $G-v \cong K_{1,2,2,2,2,2}$, which contains 120 triangles. In 
this case, $|E(G(v))| \leqslant\left(\begin{array}{l}7 \\ 2\end{array}\right)-1=20$ since $K_{1,2,2,2,2,2}$ contains no $K_{7}$-subgraph. In total, $G$ contains at most $120+20=21 n-112$ triangles (since $n=12$ ), as claimed. Now assume that $G$ has minimum degree at least 8 .

Case 5. $G$ has a separation $\left(G_{1}, G_{2}\right)$ of order at most 5: That is, $G=G_{1} \cup G_{2}$ and $G \neq G_{1}$ and $G \neq G_{2}$ and $\left|V\left(G_{1} \cap G_{2}\right)\right| \leqslant 5$. Let $n_{i}:=\left|V\left(G_{i}\right)\right|$. Since each vertex in $G_{1}-V\left(G_{2}\right)$ has degree at least $8, n_{1} \geqslant 9$. Similarly $n_{2} \geqslant 9$. By induction, $\operatorname{cliques}\left(G_{i}, 3\right) \leqslant 21 n_{i}-111$. Since $\operatorname{cliques}(G, 3) \leqslant \operatorname{cliques}\left(G_{1}, 3\right)+\operatorname{cliques}\left(G_{2}, 3\right)$,

$$
\text { cliques }(G, 3) \leqslant 21\left(n_{1}+n_{2}\right)-222 \leqslant 21(n+5)-222=21 n-117<21 n-112,
$$

as desired. Now assume that $G$ is 6-connected.

Say $G$ has $m$ edges. By Lemma 15 below, $G$ contains at most $4 m-7 n$ triangles, which is at most $4(7 n-28)-7 n=21 n-112$ by Theorem 12 (which is applicable since $G$ is neither a $\left(K_{1,2,2,2,2,2}, 6\right)$-cockade nor isomorphic to $\left.K_{2,2,2,3,3}\right)$.

Lemma 15. Every 6-connected $n$-vertex $m$-edge $K_{9}$-minor-free graph contains at most $4 m-7 n$ triangles.

Proof. First suppose $G$ contains a vertex $v$ with $G(v)$ isomorphic to a $\left(K_{2,2,2,2,2}, 5\right)$ cockade.

Say $G-N[v]$ is empty. By Lemma $6, G(v)$ has $6 n-26$ edges and $14 n-74$ triangles. Thus $G$ has $m=7 n-27$ edges and $20 n-100$ triangles. Since $20 n-100 \leqslant 4(7 n-27)-7 n$, we are done. Now assume that $G-N[v]$ is not empty. Let $C$ be a connected component of $G-N[v]$. Then $N(C) \subseteq N(v)$.

If $N(C)$ is a clique, then $|N(C)| \leqslant 5$ (since no $\left(K_{2,2,2,2,2}, 5\right)$-cockade contains $\left.K_{6}\right)$ and $G$ is not 6-connected, which is a contradiction. Thus $N(C)$ contains two non-adjacent vertices $x$ and $y$. Let $G^{\prime}$ be obtained from $G$ by contracting $C$ to a vertex $z$ and then contracting $z x$. Then $x y$ is an edge of $G^{\prime}$. Since every $\left(K_{2,2,2,2,2}, 5\right)$-cockade is edgemaximal with no $K_{8}$-minor, $G^{\prime}[N(v)]$ contains a $K_{8}$-minor, and (with $v$ ) $G^{\prime}$ contains a $K_{9}$-minor. Hence $G$ contains a $K_{9}$-minor, which is a contradiction.

Now assume that $G(v)$ is isomorphic to a $\left(K_{2,2,2,2,2}, 5\right)$-cockade for no vertex $v$. Send a charge of $\frac{1}{3}$ from each triangle to each of the three vertices in it. Each vertex $v$ receives a charge equal to $\frac{1}{3}|E(G(v))|$, which is at most $2 \operatorname{deg}(v)-7$ by Theorem 9 (which is applicable since $\operatorname{deg}(v) \geqslant 6$ and $G(v)$ is $K_{8}$-minor-free). The number of triangles, which equals the total charge, is at most $\sum_{v}(2 \operatorname{deg}(v)-7)=4 m-7 n$, as desired.

Theorem 16. The maximum number of cliques in a $K_{9}$-minor-free graph on $n \geqslant 7$ vertices equals $128(n-6)$.

Proof. Let $G$ be a $K_{9}$-minor-free graph on $n \geqslant 7$ vertices.

First suppose that $G$ is isomorphic to a $\left(K_{1,2,2,2,2,2}, 6\right)$-cockade. Note that $\operatorname{cliques}\left(K_{1,2,2,2,2,2}\right)=2 \cdot 3^{5}=486=\frac{422}{5}(11-6)+2^{6}$. By Lemma 5 with $a=\frac{422}{5}$ and $r=6$, every $n$-vertex $\left(K_{1,2,2,2,2,2}, 6\right)$-cockade contains $\frac{422}{5}(n-6)+64 \leqslant 128(n-6)$ cliques. Now assume that $G$ is isomorphic to no $\left(K_{1,2,2,2,2,2}, 6\right)$-cockade.

Now suppose that $G \cong K_{2,2,2,3,3}$. Then $\operatorname{cliques}(G)=3^{3} \cdot 4^{2}=432<128 \cdot 6=128(n-6)$. Now assume that $G \nsubseteq K_{2,2,2,3,3}$. 
By Theorem 12 and Theorem 13 and Theorem 14, we have cliques $(G, k) \leqslant\left(\begin{array}{c}7 \\ k-1\end{array}\right)(n-$ $\left.\frac{8(k-1)}{k}\right)$ for $k \in\{1,2, \ldots, 8\}$. Since cliques $(G, 0)=1$, we have cliques $(G) \leqslant 128(n-6)$.

\section{Total Number of Cliques in $\boldsymbol{K}_{t}$-minor-free Graphs}

This section considers the total number of cliques in $K_{t}$-minor-free graphs for arbitrary $t$. Recall that cliques $(n, t)$ is the maximum number of cliques in a $K_{t}$-minor-free graph on $n$ vertices. The best lower bound on $\operatorname{cliques}(n, t)$ is due to Wood [35], who observed that $K_{c \times 2}$ contains no $K_{t}$-minor where $t=\left\lfloor\frac{3}{2} c\right\rfloor+1$. Thus

$$
\operatorname{cliques}(n, t) \geqslant \operatorname{cliques}\left(K_{c \times 2}\right)=3^{c}=2^{2\left(\log _{2} 3\right) t / 3-o(t)} n \geqslant 2^{1.0566 t-o(t)} n .
$$

Upper bounds on cliques $(n, t)$ have been intensely studied over the past ten years, culminating in the recent upper bound by Fox and Wei [14] that matches the lower bound in (5) up to a lower order term. These results are summarised in the following table.

\begin{tabular}{ll}
\hline cliques $(n, t) \leqslant$ & reference \\
\hline$(c t \sqrt{\log t})^{t} n$ & Norine et al. [26] \\
$2^{c t \sqrt{\log t} n}$ & Reed and Wood [28] \\
$2^{c t \log \log t} n$ & Fomin et al. [13] \\
$2^{50 t} n$ & Lee and Oum [23] \\
$2^{5 t+o(t)} n$ & Lee and Oum [23] \\
$2^{2\left(\log _{2} 3\right) t / 3+o(t)} n$ & Fox and Wei [14] \\
\hline
\end{tabular}

Note that several authors have also studied the maximum number of cliques in graphs excluding a given subdivision [15, 23] or immersion [15].

The remainder of this section considers the following question: what is the maximum integer $t_{0}$ such that cliques $(n, t)=2^{t-2}(n-t+3)$ for all $t \leqslant t_{0}$ (thus matching the lower bound in (3))? Theorem 3 shows that $t_{0} \geqslant 9$.

Given that $K_{c \times 2}$ provides an essentially tight lower bound on cliques $(n, t)$, we now examine complete multipartite graphs in more detail. Consider a complete $c$-partite graph $G=K_{n_{1}, \ldots, n_{c}}$ where $n_{1} \geqslant \ldots \geqslant n_{c} \geqslant 1$ and $c \geqslant 2$. Then $n=\sum_{i=1}^{c} n_{i}$ is the number of vertices. Wood [35] proved that $G$ contains no $K_{t}$-minor, where

$$
t=\min \left\{\left\lfloor\frac{1}{2}(n+c)\right\rfloor+1, n-n_{1}+2\right\},
$$

and a $K_{t-1}$-minor in $G$ can be obtained from a $K_{c}$ subgraph by contracting a maximum matching in the remaining graph. If $\left\lfloor\frac{1}{2}(n+c)\right\rfloor \leqslant n-n_{1}+1$ then we say $G$ is balanced, otherwise $G$ is unbalanced (in which case the largest colour class is 'very' large). First suppose that $G$ is unbalanced. Let $m:=\frac{n-n_{1}}{c-1}$ be the average size of a colour class except the largest colour class. Then $m \geqslant 1$ and $m+1 \leqslant 2^{m}$. Thus

$$
\operatorname{cliques}(G)=\prod_{i=1}^{c}\left(n_{i}+1\right) \leqslant\left(n_{1}+1\right)(m+1)^{c-1} \leqslant\left(n_{1}+1\right) 2^{m(c-1)}=(n-t+3) 2^{t-2} .
$$


That is, every unbalanced complete multipartite graph satisfies the bound. Now consider the case in which $G$ is balanced. Let $m:=\frac{n}{c}$ be the average size of a colour class. Then $t-2 \geqslant \frac{1}{2}(n+c-1)-1=\frac{1}{2}(c(m+1)-3)$ and $n-t+3 \geqslant n-\frac{1}{2}(n+c)+3=\frac{1}{2}(n-c)+3=$ $\frac{1}{2} c(m-1)+3$. Assume that $m \geqslant 3$. Then $(m+1)^{2} \leqslant 2^{m+1}$ and $(m+1)^{c} \leqslant 2^{c(m+1) / 2}$. Since $\frac{1}{2} c(m-1)+3>2^{3 / 2}$,

$$
2^{3 / 2}(m+1)^{c} \leqslant 2^{c(m+1) / 2}\left(\frac{1}{2} c(m-1)+3\right) \leqslant 2^{c(m+1) / 2}(n-t+3)
$$

and

$$
\operatorname{cliques}(G)=\prod_{i=1}^{c}\left(n_{i}+1\right) \leqslant(m+1)^{c} \leqslant 2^{(c(m+1)-3) / 2}(n-t+3) \leqslant 2^{t-2}(n-t+3) .
$$

That is, balanced complete multipartite graphs with an average of at least three vertices per colour class satisfy the bound. Thus, if a complete multipartite graph has more than $2^{t-2}(n-t+3)$ cliques, then it is balanced and has an average of less than three vertices per colour class. This is why $K_{c \times 2}$ is a critical example. Computer search establishes that for $t \leqslant 49$ every such complete multipartite graph has at most $2^{t-2}(n-t+3)$ cliques, but for $t \geqslant 50$ there is a value of $c$ such that $K_{c \times 2}$ or $K_{1, c \times 2}$ or $K_{1,1, c \times 2}$ has no $K_{t}$-minor and contains more than $2^{t-2}(n-t+3)$ cliques. Indeed $K_{c \times 2}$ satisfies this property for $t \geqslant 62$. We therefore make the following conjecture.

Conjecture 17. $\operatorname{cliques}(n, t)=2^{t-2}(n-t+3)$ if and only if $t \leqslant 49$.

\section{$7 \quad$ Number of $k$-Cliques in $\boldsymbol{K}_{t}$-minor-free Graphs}

This section considers the maximum number of $k$-cliques in $K_{t}$-minor-free graphs. First note that Theorem 1 fails badly for large $t$. In particular, Kostochka [21, 22] and de la Vega [7] (based on the work of Bollobás et al. [3]) proved that cliques $(n, t, 2) \geqslant c_{1} t \sqrt{\log t} n$ for some constant $c_{1}>0$. Conversely, Kostochka [21, 22] and Thomason [32] proved that $\operatorname{cliques}(n, t, 2) \leqslant c_{2} t \sqrt{\log t} n$ for some constant $c_{2}>0$. Later, Thomason [33] proved that cliques $(n, t, 2)=(\alpha+o(1)) n t \sqrt{\ln t}$, where $\alpha \approx 0.319$ is precisely determined.

A graph is $d$-degenerate if every subgraph has minimum degree at most $d$. Wood [35] determined the maximum total number of cliques in a $d$-degenerate graph. Essentially the same proof determines the maximum number of $k$-cliques.

Lemma 18. For every d-degenerate graph $G$ with $n \geqslant d+1$ vertices,

$$
\operatorname{cliques}(G, k) \leqslant\left(\begin{array}{c}
d \\
k-1
\end{array}\right)\left(n-\frac{(k-1)(d+1)}{k}\right) .
$$

Proof. We proceed by induction on $n$. For the base case with $n=d+1$, the number of $k$-cliques is at most $\left(\begin{array}{l}n \\ k\end{array}\right)=\left(\begin{array}{c}d \\ k-1\end{array}\right)\left(n-\frac{k-1}{k}(d+1)\right)$. Let $G$ be a $d$-degenerate graph with $n \geqslant d+2$ vertices. There is a vertex $v$ of degree at most $d$ in $G$. The number of $k$-cliques containing $v$ is at most $\left(\begin{array}{c}d \\ k-1\end{array}\right)$. The number of $k$-cliques not containing $v$ (that is, in $G-v$ ) is at most $\left(\begin{array}{c}d \\ k-1\end{array}\right)\left(n-1-\frac{k-1}{k}(d+1)\right)$ by induction (since $G-v$ is also $d$-degenerate). In total, the number of $k$-cliques is at most $\left(\begin{array}{c}d \\ k-1\end{array}\right)\left(n-\frac{k-1}{k}(d+1)\right)$. 
The bound in Lemma 18 is tight for $d$-trees. The above-mentioned results of Kostochka $[21,22]$ and Thomason $[32,33]$ show that $K_{t}$-minor-free graphs are $c t \sqrt{\log t}$-degenerate for some constant $c$. Lemma 18 thus implies

$$
\operatorname{cliques}(t, n, k) \leqslant\left(\begin{array}{c}
c t \sqrt{\log t} \\
k-1
\end{array}\right) n \leqslant(c t \sqrt{\log t})^{k-1} n .
$$

For fixed $k$, this bound is tight up to a constant factor as we now explain. Bollobás et al. [3] proved that for a suitable constant $c>0$ and for large $t$, a random graph on $n=c t \sqrt{\log t}$ vertices has no $K_{t}$-minor with high probability. Here each edge is chosen independently

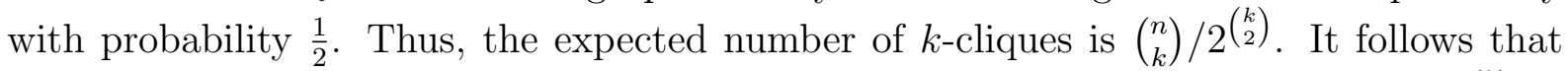
for large $t$, there exists an $n$-vertex graph with no $K_{t}$-minor and with at least $\left(\begin{array}{l}n \\ k\end{array}\right) / 2^{2}\left(\begin{array}{l}k \\ 2\end{array}\right) k$ cliques. Note that $\left(\begin{array}{l}n \\ k\end{array}\right) / 2^{\left(\begin{array}{c}k \\ 2\end{array}\right)}=c^{\prime}(t \sqrt{\log t})^{k-1} n$ for a suitable constant $c^{\prime}$. Thus there exists an $n$-vertex graph $G$ with no $K_{t}$-minor such that cliques $(G, k) \geqslant c^{\prime}(t \sqrt{\log t})^{k-1} n$. Taking disjoint copies of $G$ gives a graph with the same property, where $n \gg t$. Summarising, for fixed $k$, there are constants $c_{1}$ and $c_{2}$ such that

$$
c_{1}(t \sqrt{\log t})^{k-1} n \leqslant \operatorname{cliques}(n, t, k) \leqslant c_{2}(t \sqrt{\log t})^{k-1} n .
$$

Thus cliques $(n, t, k)$ is determined up to a constant factor for fixed $k$. But as $k$ increases with $t$, determining cliques $(n, t, k)$ is wide open. First note that a random graph will have few large cliques. In fact, the size of the largest clique in a random graph on $t$ vertices is sharply concentrated around $2 \log _{2} t[4,25]$. This motivates the following conjecture about 'large' cliques in $K_{t}$-minor-free graphs.

Conjecture 19. For some constants $c_{1}, c_{2}>0$, for all integers $t \geqslant 3$ and $k \geqslant c_{1} \log t$ and $n \geqslant t-1$,

$$
\text { cliques }(n, t, k) \leqslant\left(c_{2} t\right)^{k} n \text {. }
$$

This conjecture is true for $k \geqslant \frac{2\left(\log _{2} 3\right) t / 3+o(t)}{\log _{2} t}$, since the above-mentioned upper bound of Fox and Wei [14] implies:

$$
\operatorname{cliques}(n, t, k) \leqslant \operatorname{cliques}(n, t) \leqslant 2^{2\left(\log _{2} 3\right) t / 3+o(t)} n \leqslant t^{k} n \text {. }
$$

For very large cliques in $K_{t}$-minor-free graphs, we conjecture that the lower bound in (2) is tight.

Conjecture 20. For some $\lambda \in\left[\frac{1}{3}, 1\right)$, for all integers $t \geqslant 3$ and $k \geqslant \lambda t$ and $n \geqslant t-1$,

$$
\operatorname{cliques}(n, t, k) \leqslant\left(\begin{array}{c}
t-2 \\
k-1
\end{array}\right)\left(n-\frac{(k-1)(t-1)}{k}\right) .
$$

We now provide two pieces of evidence in support of this conjecture. First, even though $K_{c \times 2}$ contains many cliques in total (see (5)), it contains no clique of order $c+1 \approx \frac{2}{3} t$, implying $K_{c \times 2}$ satisfies Conjecture 20 for $\lambda=\frac{2}{3}$. Our second piece of evidence is to prove Conjecture 20 for $k=t-1$ (which is the largest non-trivial value of $k$ ). 
Proposition 21. For all integers $t \geqslant 2$ and $n \geqslant t-1$,

$$
\operatorname{cliques}(n, t, t-1)=n-t+2 \text {. }
$$

Proof. The lower bound is provided by (2). For the upper bound, we proceed by induction on $n$. Let $G$ be a $K_{t}$-minor-free graph on $n \geqslant t-1$ vertices. If $n=t-1$ then $\operatorname{cliques}(G, t-$ $1) \leqslant 1$ as desired. Now assume that $n \geqslant t$. We may assume that $G$ contains $K_{t-1}$. Let $K$ be the vertex set of a copy of $K_{t-1}$ in $G$. Let $X_{1}, \ldots, X_{r}$ be the vertex sets of the connected components of $G-K$. If for some $i \in[1, r]$, every vertex in $K$ has a neighbour in $X_{i}$, then contracting $G\left[X_{i}\right]$ to a single vertex (with $K$ ) gives a $K_{t}$-minor in $G$. Thus, for each $i \in[1, r]$, some vertex $v_{i}$ in $K$ is adjacent to no vertex in $X_{i}$. Each copy of $K_{t-1}$ distinct from $K$ is contained in $G_{i}:=G\left[\left(X_{i} \cup K\right) \backslash\left\{v_{i}\right\}\right]$ for some $i \in[1, r]$. Thus

$$
\operatorname{cliques}(G, t-1)=1+\sum_{i=1}^{r} \operatorname{cliques}\left(G_{i}, t-1\right) .
$$

Note that each $G_{i}$ has $\left|X_{i}\right|+t-2$ vertices, which is at least $t-1$ and less than $n$. By induction,

$$
\operatorname{cliques}(G, t-1) \leqslant 1+\sum_{i=1}^{r}\left|X_{i}\right|=1+n-(t-1)=n-t+2 \text {. }
$$

Finally we justify the lower bound of $\lambda \geqslant \frac{1}{3}$ in Conjecture 20. Again, $K_{c \times 2}$ is the example. Assume $c$ is even. As noted earlier, $G$ contains no $K_{t}$-minor where $t=\frac{3}{2} c+1$. Observe that cliques $\left(K_{c \times 2}, k\right)=\left(\begin{array}{l}c \\ k\end{array}\right) 2^{k}$. The following series of conditions are equivalent.

$$
\begin{aligned}
\operatorname{cliques}\left(K_{c \times 2}, k\right) & \leqslant\left(\begin{array}{l}
t-2 \\
k-1
\end{array}\right)\left(n-\frac{(k-1)(t-1)}{k}\right) \\
\left(\begin{array}{l}
c \\
k
\end{array}\right) 2^{k} & \leqslant\left(\begin{array}{l}
t-2 \\
k-1
\end{array}\right)\left(2 c-\left(1-\frac{1}{k}\right) \frac{3 c}{2}\right) \\
\frac{c ! 2^{k}}{(c-k) ! k !} & \leqslant \frac{(t-2) !}{(k-1) !(t-k-1) !}\left(\frac{c}{2}+\frac{3 c}{2 k}\right) \\
\frac{c ! 2^{k+1}}{(c-k) !} & \leqslant \frac{(t-2) ! c(k+3)}{(t-k-1) !} \\
2^{k+1} \prod_{i=1}^{k-1}(c-i) & \leqslant(k+3) \prod_{i=1}^{k-1}(t-i-1) \\
2^{k+1} \prod_{i=1}^{k-1}(c-i) & \leqslant(k+3) \prod_{i=1}^{k-1}\left(\frac{3 c}{2}-i\right)
\end{aligned}
$$

Thus $K_{c \times 2}$ satisfies Conjecture 20 for a particular value of $k$ if and only if (6) holds. We now show that (6) is not satisfied by $K_{c \times 2}$ for small $k$; that is, $K_{c \times 2}$ has many small 
cliques. Fix $\epsilon>0$. Let $k \geqslant k(\epsilon)$ and $c \geqslant(2+\epsilon) k$. Since $1 \leqslant i<k \leqslant \frac{c}{2+\epsilon}$, it follows that $2(c-i) \geqslant\left(1+\frac{\epsilon}{4+3 \epsilon}\right)\left(\frac{3 c}{2}-i\right)$. Thus

$$
2^{k+1} \prod_{i=1}^{k-1}(c-i)>4\left(1+\frac{\epsilon}{4+3 \epsilon}\right)^{k-1} \prod_{i=1}^{k-1}\left(\frac{3 c}{2}-i\right) .
$$

Now $4\left(1+\frac{\epsilon}{4+3 \epsilon}\right)^{k-1}>k+3$ for large $k \geqslant k(\epsilon)$. Thus (6) is not satisfied, and

$$
\operatorname{cliques}\left(K_{c \times 2}, k\right)>\left(\begin{array}{c}
t-2 \\
k-1
\end{array}\right)\left(n-\frac{(k-1)(t-1)}{k}\right) .
$$

Thus $k>\frac{c}{2+\epsilon}$ for Conjecture 20 to hold for $K_{c \times 2}$. Since $c \approx \frac{2}{3} t$, this says that $\lambda \geqslant \frac{1}{3}-\epsilon$ in Conjecture 20.

\section{Acknowledgements}

Many thanks to both referees for their very thorough reviews.

\section{References}

[1] James Alexander, Jonathan Cutler, And Tim Mink. Independent sets in graphs with given minimum degree. Electron. J. Combin., 19(3):Paper 37, 11, 2012. http://www.combinatorics.org/ojs/index.php/eljc/article/view/ v19i3p37. MR: 2988859.

[2] Noga Alon and Raphael Yuster. Matrix sparsification and nested dissection over arbitrary fields. J. ACM, 60(4):Art. 25, 18, 2013. doi:10.1145/2508028.2505989. MR:3116510.

[3] Béla Bollobás, Paul A. Catlin, and Paul Erdős. Hadwiger's conjecture is true for almost every graph. European J. Combin., 1(3):195-199, 1980. doi:10.1016/S0195-6698(80)80001-1. MR: 593989.

[4] Béla Bollobás and Paul Erdős. Cliques in random graphs. Math. Proc. Cambridge Philos. Soc., 80(3):419-427, 1976. doi:10.1017/S0305004100053056. MR: 0498256.

[5] Jonathan Cutler and A. J. Radcliffe. Extremal problems for independent set enumeration. Electron. J. Combin., 18(1):Paper 169, 17, 2011. MR: 2831105.

[6] Jonathan Cutler and A. J. Radcliffe. The maximum number of complete subgraphs in a graph with given maximum degree. J. Combin. Theory Ser. B, 104:6071, 2014. doi:10.1016/j.jctb.2013.10.003. MR: 3132744

[7] W. Fernandez DE LA Vega. On the maximum density of graphs which have no subcontraction to $K^{s}$. Discrete Math., 46(1):109-110, 1983. doi:10.1016/0012-365X (83) 90280-7. MR: 0708172. 
[8] Gabriel A. Dirac. Homomorphism theorems for graphs. Math. Ann., 153:69-80, 1964. doi:10.1007/BF01361708. MR:0160203.

[9] Vida Dujmović, Gašper Fijavž, Gwenä̈l Joret, Thom Sulanke, AND DAVID R. WOOD. On the maximum number of cliques in a graph embedded in a surface. European J. Combin., 32(8):1244-1252, 2011. doi:10.1016/j.ejc.2011.04.001. MR:2838013.

[10] JÜrgen ECKhoff. The maximum number of triangles in a $K_{4}$-free graph. Discrete Math., 194(1-3):95-106, 1999. doi:10.1016/S0012-365X(98)00120-4. MR: 1654980 .

[11] Jürgen Eckhoff. A new Turán-type theorem for cliques in graphs. Discrete Math., 282(1-3):113-122, 2004. doi:10.1016/j.disc.2003.11.007. MR: 2059512.

[12] John Engbers And David Galvin. Counting independent sets of a fixed size in graphs with a given minimum degree. J. Graph Theory, 76(2):149-168, 2014. doi:10.1002/jgt.21756. MR: 3190271

[13] Fedor V. Fomin, Sang il Oum, and Dimitrios M. Thilikos. Rank-width and tree-width of $H$-minor-free graphs. European J. Combin., 31(7):1617-1628, 2010. doi:10.1016/j.ejc.2010.05.003. MR: 2673004.

[14] JACOB FOX AND FAN WEI. On the number of cliques in graphs with a forbidden minor. 2016. arXiv: 1603.07056.

[15] JaCOB Fox AND FAN WeI. On the number of cliques in graphs with a forbidden subdivision or immersion. 2016. arXiv: 1606.06810.

[16] Andrew Frohmader. A Kruskal-Katona type theorem for graphs. J. Combin. Theory Ser. A, 117(1):17-37, 2010. doi:10.1016/j.jcta.2009.04.003. MR: 2391144.

[17] David Galvin. Two problems on independent sets in graphs. Discrete Math., 311(20):2105-2112, 2011. doi:10.1016/j.disc.2011.06.015. MR: 2825652.

[18] Wenying Gan, Po-Shen Loh, and Benny Sudakov. Maximizing the number of independent sets of a fixed size. Combin. Probab. Comput., 24(3):521-527, 2015. doi:10.1017/S0963548314000546. MR: 3326430.

[19] S. Louis Hakimi and Edward F. Schmeichel. On the number of cycles of length $k$ in a maximal planar graph. J. Graph Theory, 3(1):69-86, 1979. doi:10.1002/jgt.3190030108. MR: 519175.

[20] Leif K. Jørgensen. Contractions to K. J. Graph Theory, 18(5):431-448, 1994. doi:10.1002/jgt.3190180502. MR: 1283309.

[21] Alexandr V. Kostochka. The minimum Hadwiger number for graphs with a given mean degree of vertices. Metody Diskret. Analiz., 38:37-58, 1982. MR:0713722.

[22] Alexandr V. Kostochka. Lower bound of the Hadwiger number of graphs by their average degree. Combinatorica, 4(4):307-316, 1984. doi:10.1007/BF02579141. MR: 0779891. 
[23] Choongbum Lee And Sang-IL Oum. Number of cliques in graphs with a forbidden subdivision. SIAM J. Disc. Math., 29(4):1999-2005, 2015. doi:10.1137/140979988. MR: 3414466

[24] Wolfgang Mader. Homomorphiesätze für Graphen. Math. Ann., 178:154-168, 1968. doi:10.1007/BF01350657. MR:0229550.

[25] David W. Matula. On the complete subgraphs of a random graph. In Proc. Second Chapel Hill Conf. on Combinatorial Mathematics and its Applications, pp. 356-369. Univ. North Carolina, U.S.A., 1970. MR: 0266796.

[26] Serguei Norine, Paul Seymour, Robin Thomas, and Paul Wollan. Proper minor-closed families are small. J. Combin. Theory Ser. B, 96(5):754-757, 2006. doi:10.1016/j.jctb.2006.01.006. MR:2236510.

[27] Christos H. Papadimitriou and Mihalis Yannakakis. The clique problem for planar graphs. Inform. Process. Lett., 13(4-5):131-133, 1981. doi:10.1016/0020-0190(81)90041-7. MR:0651460.

[28] Bruce Reed and David R. Wood. A linear time algorithm to find a separator in a graph excluding a minor. ACM Transactions on Algorithms, 5(4):\#39, 2009. doi:10.1145/1597036.1597043. MR: 2571902.

[29] Igor Rivin. Counting cycles and finite dimensional $L^{p}$ norms. Adv. in Appl. Math., 29(4):647-662, 2002. doi:10.1016/S0196-8858(02)00037-4. MR: 1943370.

[30] Zi-Xia Song and Robin Thomas. The extremal function for $K_{9}$ minors. $J$. Combin. Theory Ser. B, 96(2):240-252, 2006. doi:10.1016/j.jctb.2005.07.008. MR: 2208353.

[31] Siamak Tazari and Matthias Müller-Hannemann. Shortest paths in linear time on minor-closed graph classes, with an application to Steiner tree approximation. Discrete Appl. Math., 157(4):673-684, 2009. doi:10.1016/j.dam.2008.08.002. MR: 2499480

[32] Andrew Thomason. An extremal function for contractions of graphs. Math. Proc. Cambridge Philos. Soc., 95(2):261-265, 1984. doi:10.1017/S0305004100061521. MR: 0735367.

[33] Andrew Thomason. The extremal function for complete minors. J. Combin. Theory Ser. B, 81(2):318-338, 2001. doi:10.1006/jctb.2000.2013. MR: 1814910.

[34] Paul Turán. On an extremal problem in graph theory. Mat. Fiz. Lapok, 48:436-452, 1941.

[35] David R. Wood. On the maximum number of cliques in a graph. Graphs Combin., 23(3):337-352, 2007. doi:10.1007/s00373-007-0738-8. MR:2320588.

[36] Alexander A. Zykov. On some properties of linear complexes. Mat. Sbornik N.S., 24(66):163-188, 1949. MR: 0035428. 